\title{
Recurrence without uniform recurrence
}

\author{
T W KORNER \\ Trinity Hall, University of Cambridge, Cambridge, England
}

(Recelved 10 November 1986)

\begin{abstract}
We construct a minımal homeomorphism of a compact space such that a sequence of its iterates converges pointwise to the identity but no sequence of its iterates converges uniformly
\end{abstract}

\section{Introduction}

The object of this paper is to construct the system described below

THEOREM 11 There exists a compact, complete, metric space $(X, d)$ and a homeomorphism $T X \rightarrow X$ such that

(1) the set $\left\{T^{n} x \quad n \geq 0\right\}$ is dense in $X$ for each $x \in X$,

(11) $\sup _{x \in X} d\left(T^{m} x, x\right) \geq 1$ for each $m \geq 1$, yet

(111) there exists a sequence $n(1)<n(2)<\quad$ such that $d\left(T^{n(\jmath)} x, x\right) \rightarrow 0$ as $j \rightarrow \infty$ for each $x \in X$

I should like to thank B Weiss for suggesting this problem and for pointing out a flaw in my original attack Since, in my opınıon, the interest of this paper hes more in the method of construction than in the result itself I shall begin by indicating why we might expect such a construction to be fairly complicated The following result was pointed out to me, again by B Weiss

Lemma 12 Suppose that $(X, d)$ is a compact metric space and $T X \rightarrow X$ is a homeomorphism such that $\left\{T^{n} x \quad n \geq 0\right\}$ is dense in $X$ for each $x \in X$ Suppose further that we can find a sequence $n(1)<n(2)<\quad$ of integers and a sequence $\varepsilon(1)>$ $\varepsilon(2)>\quad$ of positive real numbers such that $\varepsilon(j) \rightarrow 0$ as $\jmath \rightarrow \infty$ and such that for each $x \in X$ there exists an integer $j_{0}(x)$ with

$$
d\left(T^{n(\jmath)} x, x\right) \leq \varepsilon(\jmath) \quad \text { for all } J \geq J_{0}(x)
$$

Then $\sup _{x \in X} d\left(T^{n(j)} x, x\right) \rightarrow 0$ as $j \rightarrow \infty$

Proof Let

$$
E(k)=\left\{x \in X \quad d\left(T^{n(J)} x, x\right) \leq \varepsilon(J) \text { for all } J \geq k\right\}
$$

Then each $E(k)$ is closed and, by hypothesis, $\bigcup_{k=1}^{\infty} E(k)=X$ By the Bare category theorem we can find a $k_{0}$ and a non-empty open set $U$ such that $U \subseteq E\left(k_{0}\right)$ Since $\left\{T^{n} x \quad n \geq 0\right\}$ is dense in $X$, the sets $T^{-n} U[n \geq 0]$ form an open cover of $X$ and so, by compactness, we can find an $M$ such that $\bigcup_{m=0}^{M} T^{-m} U=X, 1$ e such that for each $x \in X$ there exists an $0 \leq m \leq N$ with $T^{m} x \in U$ 
Now let $\omega(\varepsilon)$ be the common modulus of contınuity of $I, T^{-1}, T^{-2}, \quad, T^{-M}, 1 \mathrm{e}$ let

$$
\omega(\varepsilon)=\sup _{0 \leq m \leq N} \sup \left\{d\left(T^{-m} u, T^{-m} v\right) d(u, v) \leq \varepsilon\right\}
$$

We observe that $\omega(\varepsilon) \rightarrow 0$ as $\varepsilon \rightarrow 0$ and that we can now conclude the proof For if $x \in X$ then $T^{m} x \in U$ for some $0 \leq m \leq M$ and so, if $k \geq k_{0}$

whence

$$
d\left(T^{n(k)} T^{m} x, T^{m} x\right) \leq \varepsilon(k)
$$

$$
d\left(T^{n(k)} x, x\right)=d\left(T^{-m}\left(T^{n(k)} T^{m} x\right), T^{-m}\left(T^{m} x\right)\right) \leq \varepsilon(k)
$$

Thus $\sup _{x \in X} d\left(T^{n(k)} x, x\right) \leq \omega(\varepsilon(k))$ for all $k \geq k_{0}$ and the lemma follows

\section{Reduction to a semi-combinatorial problem}

Let $\Lambda$ be a compact subset of $\mathbb{R}$ and let $\Lambda^{\mathbb{Z}}$ be the space of two-sided sequences $x \mathbb{Z} \rightarrow \Lambda^{\mathbb{Z}}$ Let $d$ be the distance defined on $\Lambda^{\mathbb{Z}}$ by

$$
d(x, y)=\sup _{k \in \mathbb{Z}} 2^{-|k|}\left|x_{k}-y_{k}\right|
$$

and let $T$ be the shift map $T \Lambda^{\mathbb{Z}} \rightarrow \Lambda^{\mathbb{Z}}$ given by

$$
(T x)=x_{k+1} \quad(k \in \mathbb{Z})
$$

Then $\left(\Lambda^{\mathbb{Z}}, d\right)$ is a complete, compact, metric space and $T$ a homeomorphism Furstenberg and his collaborators Katznelson and Weiss have brilliantly exploited the analytic structure of $\left(\Lambda^{z}, d, T\right)$ to obtain results on the combinatorial structure of $\Lambda^{\mathbb{Z}}$ (see [1]) We shall reverse the process by using a combinatorial construction in $\Lambda^{\mathbb{Z}}$ to obtain the analytic Theorem 11 Examples of such constructions are discussed in [1, Chapter $1, \S \S 3,5]$ The examples given there take $\Lambda$ to be a finite set but we shall use $\Lambda=[-1,1]$ The fact that $\Lambda$ is then connected is essential for our construction

From now on $T$ and $d$ will have the meanings assigned to them in the previous paragraph We intend to deduce Theorem 11 from the following lemma which is not yet completely combinatorial since it mentions metric closure

LEMMA 21 There exists a subset $X$ of $[-1,1]^{\mathbb{Z}}$ and a sequence $n(1)<n(2)<$ with the following properties

(1) If $x, y \in X$ then given $k \geq 1$ and $\varepsilon>0$ we can find an $m \geq 1$ such that $\left|y_{m+r}-x_{r}\right| \leq$ $\varepsilon$ for all $|r| \leq k$

(11) For each $m \neq 0$ there exists an $x \in X$ such that $\left|x_{m}-x_{0}\right| \geq 1$

(111) If $x \in X$ and $p \geq k+1$ then, for each $\imath \in \mathbb{Z}$,

for all $p-1 \geq l \geq k$

$$
\left|x_{t}-x_{i+n(p)}\right|>2^{2-k} \Rightarrow\left|x_{1}-x_{i+n(l)}\right| \leq 2^{2-I}
$$

(1v) If $x \in X$ then $T x, T^{-1} x \in X$

(v) $X$ is closed in $\left([-1,1]^{\mathrm{Z}}, d\right)$

Proof of Theorem 11 from Lemma 21 Condition (v) tells us that $(X, d)$ is complete and compact and condition (iv) shows us that $T$ (restricted to $X$ ) is a homeomorphism Conditions ( 1 ), (11) and (111) yield the corresponding conditions of the theorem Thus if $x, y \in X$ and $\varepsilon>0$ then, choosing $k \geq 1$ with $2^{k}>\varepsilon^{-1}$, condition (1) tells us 
that we can find an $m \geq 1$ such that $\left|y_{m+r}-x_{r}\right| \leq \varepsilon$ for all $|r| \leq k$ and so

$$
d\left(T^{m} y, x\right)=\sup _{r \in \mathbb{Z}} 2^{-|r|}\left|y_{m+r}-x_{r}\right| \leq \varepsilon
$$

Thus the orbit of each point $y$ is dense and (1) holds Similarly condition (11) tells us that for each $m$ there exists an $x \in X$ with $\left|x_{m}-x_{0}\right| \geq 1$ and so $d\left(T^{m} x, x\right) \geq 1$ Finally we observe that if $x \in X$ condition (111) implies that $\left|x_{1}-x_{1+n(p)}\right| \geq 2^{2-r}$ for at most one value of $p \geq r+1$ and so $x_{1}-x_{i+n(p)} \rightarrow 0$ as $p \rightarrow \infty$ for each $t \in \mathbb{Z}$ Thus $d\left(T^{n(p)} x, x\right) \rightarrow 0$ as $p \rightarrow \infty$ for each $x \in X$ as required

The reader should observe that, although condition (111) of the lemma forces $d\left(T^{n(p)} x, x\right) \rightarrow 0$, the convergence can have hiccups These hiccups enable us to evade the conclusion of Lemma 12 From a more combinatorial point of view our problem has been to find a condition which is weak enough to be compatible with condition (1) and (11) and yet strong enough to force pointwise convergence

\section{Reduction to a purely combinatorial problem}

The next step is in an obvious direction We show how Lemma 21 can be deduced from a purely combinatorial lemma

LEMMA 31 There exists a sequence of integers $m(J) \geq 5$ and a collection of subsets $U(J) \subseteq[-1,1]^{n(J)}$, where $n(J)=m(1) m(2) \quad m(J)$, with the following properties (we adopt the convention that if $u \in U(J)$ then $u_{l n(j)+r}=u_{r}$ for all $l \in \mathbb{Z}, 1 \leq r \leq n(J)$ )

$(1)_{j+1}$ If $u \in U(\jmath)$ and $v \in U(\jmath+1)$ then we can find an $l, 1 \leq l \leq m(\jmath+1)$, such that

$$
\left|u_{r}-v_{\ln (\jmath)+r}\right| \leq 2^{-\jmath-1} \text { for all } 1 \leq r \leq n(J)
$$

(11) If $1 \leq|m| \leq n(J)-1$ we can find a $u \in U(J)$ and $1 \leq r, s \leq 2 n(J)$ such that $s-r=m$ and $\left|u_{r}-u_{s}\right| \geq 1$

(111) If $u \in U(J)$ then, for each $J-1 \geq p \geq k+1$ and each $\imath \in \mathbb{Z}$

for all $p-1 \geq l \geq k$

$$
\left|u_{i}-u_{i+n(p)}\right|>2^{2-k} \Rightarrow\left|u_{i}-u_{i+n(l)}\right| \leq 2^{2-l},
$$

(iv) ${ }_{j+1}$ If $v \in U(J+1)$ and $1 \leq l \leq m(J)$ then writtng $u_{r}=v_{\ln (J)+r}(1 \leq r \leq n(J))$ we have $u \in U(J)$

$(\mathrm{v})_{j+1}$ There exists an $e(\jmath) \in U(\jmath)$ such that, if $v \in U(\jmath+1)$ then $v_{r}=$ $v_{n(J+1)-n(\jmath)+r}=e(J)_{r}$ for all $1 \leq r \leq n(J)$

Thus the 'sentences' of $U(J+2)$ are composed of 'words' from $U(J+1)$ and these words in turn are composed of 'letters' from $U(J)$ Notice that each 'sentence' in $U(j+2)$ begins and ends with the same 'buffer word' $e(j+1)$ Thus if we study a short sequence of 'letters' in some 'paragraph' or 'chapter' we know that the sequence either lies well within a single sentence or falls within two successive copies of the same 'buffer word'

Using Lemma 31 it is very easy to prove a lemma from which Lemma 21 follows almost immediately

LEMMA 32 There extsts a sequence $n(1)<n(2)<\quad$ and a collection of subsets $X(\mathrm{~J})$ of $[-1,1]^{2}$ with the following properties

(1) If $x \in X(\jmath+1)$ and $y \in X(j+2)$ then given $1 \leq k \leq \jmath$ we can find an $m \geq 1$ such that $\left|x_{r}-y_{r+m}\right| \leq 2^{-J-2}$ for all $|r| \leq J$ 
(11) If $1 \leq|m| \leq n(\jmath)-1$ we can find an $x \in X(\jmath)$ such that $\left|x_{m}-x_{0}\right| \geq 1$

(111) If $x \in X(J)$ then, for each $J-1 \geq p \geq k+1$ and each $l \in \mathbb{Z}$

$$
\left|x_{i}-x_{i+n(p)}\right|>2^{2-k} \Rightarrow\left|x_{i}-x_{i+n(l)}\right| \leq 2^{2-l}
$$

for all $p-1 \geq l \geq k$.

(iv) If $x \in X(j)$, then $T x, T^{-1} x \in X(j)$

(v) $X(J)$ is closed in $\left([-1,1]^{\mathbb{Z}}, d\right)$

(v1) $X(\mathrm{~J}) \supseteq X(\mathrm{~J}+1)$

Proof of Lemma 32 from Lemma 31 If we replace the $U(J)$ of Lemma 31 by their closures (with respect to the usual topology on $[-1,1]^{n(J)}$ ) the conditions of that lemma still apply We may therefore take the $U(J)$ to be closed We then define $X(J)$ to be the collection of all infinite strings of words from $U(J)$ More precisely, let us say that $x \in X(q, J)$ if and only if whenever $l \in \mathbb{Z}$ and $u_{r}=x_{\ln (J)+q+r}(1 \leq r \leq n(J))$ it follows that $u \in U(J)$ We set $X(J)=\bigcup_{q=0}^{n(j)-1} X(q, j)$ We note that

(iv) $X(q, J)=X(q+n(\jmath), J)$ for all $q \in \mathbb{Z}$, and

(vi $)^{\prime} X(q, J) \subseteq X(q, J+1)$ for all $q \in \mathbb{Z}$

Conditions (iv) and (vi) follow at once Since we have taken $U(J)$ closed in $[-1,1]^{n(J)}$ it follows easily that $X(q, J)$ is closed in $\left([-1,1]^{\mathbb{Z}}, d\right)$ and condition $(\mathrm{v})$ follows

To prove (1) we observe that $x \in X(q, j+1)$ and $y \in X(p, j+2)$ where (using (iv)') we may suppose $|q| \leq n(j+1) / 2+1$ and $p \geq n(j+2)+1$ Two cases arise according as $|q|>\jmath$ or $|q| \leq \jmath$ If $|q|>\jmath$ then, writing $Q=q$ if $q<0$ and $Q=q-n(j+1)$ if $q \geq 0$, we know that if $u_{s}=x_{Q+s}[1 \leq s \leq n(j+1)]$ and $v_{t}=y_{p+t}[1 \leq t \leq n(j+2)]$ then $u \in U(\jmath+1)$ and $v \in V(J+2)$ By condition (1) of Lemma 31 we can find an $1 \leq l \leq$ $m(j+2)$ such that $\left|u_{s}-v_{\ln (J+1)+s}\right| \leq 2^{-J-2}$ for $1 \leq s \leq n(J+1)$ Thus taking $m=$ $p+\ln (j+1)-Q$ we have $m \geq 1$ and

for $|r| \leq J$

$$
\left|y_{m+r}-x_{r}\right|=\left|v_{m+p+r}-u_{r-Q}\right|=\left|v_{l n(j+1)+r-Q}-u_{r-Q}\right| \leq 2^{-J-2}
$$

If $|q| \leq \jmath$ then, observing that $y \in X(\jmath+2, p) \leq X(\jmath+1, p)$, and using condition (v) of Lemma 32 , we know that, writing $u_{s}=x_{q+s}, v_{s}=y_{p+s}[|s| \leq 2 \jmath]$ we have $u_{s}=e(\jmath)_{s}=v_{s}$ for $1 \leq s \leq 2 \jmath$ and $u_{s}=e(J)_{n(\jmath)+s}=v_{s}$ for $-2 \jmath \leq s \leq 0$ Thus $x_{r}=u_{r-q}=$ $v_{r-q}=y_{p-q-r}$ for $|r| \leq J$ and, settıng $m=p-q$ we have $m \geq 1$ and $\left|y_{m-r-x_{r}}\right|=0 \leq 2^{-J-2}$ for $|\boldsymbol{r}| \leq J$ Thus (1) holds

The proof of (111) involves a similar splittıng into cases Using (iv)', we know that $x \in X(q, J)$ with $q+1 \leq 1 \leq q+n(j)$ If $t+n(p) \leq q+n(j)$ we set $u_{r}=x_{q+r} \quad(1 \leq r \leq$ $n(j))$ so that $u \in U(j)$ Then $\left|x_{t}-x_{t+n(p)}\right|>2^{2-k}$ implies $\left|u_{1-q}-u_{t+n(p)-q}\right|>2^{2-k}$ which by Lemma 31 (111) implies $\left|u_{t-q}-u_{i+n(t)-q}\right| \leq 2^{2-l}$ and so $\left|x_{t}-x_{i+n(l)}\right| \leq 2^{2-l}$ for all $p-1 \leq l \leq k$ If, on the other hand, $l+n(p)>q+n(J)$ we know from Lemma $31(\mathrm{v})$ that

$$
x_{q+n(J)-n(J-1)+r}=e(J-1)_{r} \quad \text { for } 1 \leq r \leq 2 n(J-1)
$$

(where, by convention $e(J-1)_{n(J-1)+s}=e(J-1)_{s}(1 \leq s \leq n(J-1))$ It follows that

$$
x_{i}=e(J-1)_{i-q-n(J)+n(J-1)}=e(J-1)_{i-q-n(j)+2 n(j-1)}=x_{1+n(j-1)}
$$

Thus if $\left|x_{\imath}-x_{i+n(p)}\right|>2^{2-k}$ we have $\jmath-2 \geq p$ and

$$
\left|e(J-1)_{\imath-q-n(j)+n(j-1)}-e(J-1)_{\imath-q-n(j)+n(j-1)+n(p)}\right|>2^{2-k}
$$


whence, by Lemma $31(111)$,

$$
\left|e(J-1)_{1-q-n(\jmath)+n(j-1)}-e(J-1)_{\imath-q-n(\jmath)+n(J-1)+n(l)}\right| \leq 2^{2-l} \text {, }
$$

and so $\left|x_{1}-x_{\imath+n(l)}\right| \leq 2^{2-l}$ for all $p-1 \geq l \geq k$. Thus (111) holds

Finally, to prove (11) observe that by condition (11) of Lemma 31 we can find $u \in U(J)$ and $1 \leq r, s \leq 2 n(J)$ such that $s-r=m$ and $\left|u_{r}-u_{s}\right| \geq 1$ Setting $x_{\ln (J)+t-r}=u_{t}$ $(1 \leq t \leq n(\jmath), l \in \mathbb{Z})$ we have $x \in X(\jmath)$ and $\left|x_{0}-x_{m}\right| \geq 1$

Proof of Lemma 21 from Lemma 32 Set $X=\bigcup_{j=1}^{\infty} X(j)$ Then conditions (1), (111), (iv) and (v) of Lemma 21 follow at once from conditions (1), (111), (1v) and (v) of Lemma 32 To prove Lemma 2 1(11) we observe that by Lemma 32 (11) we can find, for each $J \geq 1$, an $x(J) \in X(J)$ such that $\left|x(J)_{m}-x(J)_{0}\right| \geq 1$ Since $\left([-1,1]^{\mathbb{Z}}, d\right)$ is compact the sequence of $x(j)$ must have a limit point $x$, say By Lemma $32(\mathrm{vi})$ $x(\jmath) \in X(k)$ for all $\jmath \geq k$ and so, since $X(k)$ 1s closed, $x \in X(k)$ for all $k \geq 1$ Thus $x \in X$ and, since $d(x(J), x) \rightarrow 0,\left|x_{m}-x_{0}\right| \geq 1$

Remark. In fact, our construction will be sufficiently explicit to allow us to write down a specific $x$ without appealing to general results

\section{The induction}

Although Lemma 31 has an inductive form it does not, as it stands, lend itself to an inductive proof The key step in the paper consists in replacing it with a narrower, more specific, result which can be obtained by induction

LEMMA 41 There exists a sequence of integers $m(J) \geq 5$ and three sequences of functions

$$
\begin{aligned}
& a(\jmath, \quad)[0,1] \rightarrow[-1,1]^{n(J)} \\
& b(J, \quad)[0,1] \rightarrow[-1,1]^{n(J)} \\
& c(J, \quad)[0,1] \rightarrow[-1,1]^{n(J)}
\end{aligned}
$$

where $n(J)=m(1) m(2) \quad m(J)$ with the following properties (we write $U(J)=$ $\{a(J, t) \quad t \in[0,1]\} \cup\{b(J, t) \quad t \in[0,1]\} \cup\{c(J, t) \quad t \in[0,1]\}$ and adopt the convention that if $u \in U(j)$ then $u_{l n(j)+r}=u_{r}$ for all $\left.l \in \mathbb{Z}, 1 \leq r \leq n(j)\right)$ that

$(1)_{j+1} \quad$ If $u \in U(J)$ and $v \in U(J+1)$ then we can find an $l, 1 \leq l \leq m(J+1)$, such

$$
\left|u_{r}-v_{\ln (J)+r}\right| \leq 2^{-\jmath-1} \quad \text { for all } 1 \leq r \leq n(\jmath)
$$

(11), If $1 \leq m \leq n(j)-1$ we can find $u \in\{a(\jmath, 1), b(J, 1), c(\jmath, 1)\}, 1 \leq r, s \leq 2 n(J)$ such that $s-r=m$ and $\left|u_{r}-u_{s}\right| \geq 1$

(111) ${ }_{j}$ If $u \in U(j)$ then for each $J-1 \geq p \geq k+1$ and each $i \in \mathbb{Z}$

$$
\left|u_{t}-t_{1+n(p)}\right|>2^{2-k} \Rightarrow\left|u_{i}-u_{i+n(l)}\right| \leq 2^{2-l}
$$

for all $p-1 \geq l \geq k$

(1v) $)_{j+1}$ If $v \in U(J+1)$ and $1 \leq l \leq m(J)$ then writting $u_{r}=v_{\ln (j)+r}(1 \leq r \leq n(j))$ we have $u \in U(\mathrm{~J})$

$(\mathrm{v})_{j+1}$ If $v \in U(\mathrm{~J}+1)$ then $v_{r}=v_{n(\jmath+1)-n(\jmath)+r}=c(\jmath, 0)_{r}$ for all $1 \leq r \leq n(J)$

(v1) $\quad a(\jmath, 0)=b(\jmath, 0)=c(\jmath, 0)$

(v11), There exists an $N(J), 1 \leq N(J) \leq n(J)$, such that $a(\jmath, t)_{N(J)}=t$ and $b(J, t)_{N(1)}=-t$ 
(v111), If $1 \leq k \leq J-1,1 \leq \imath \leq n(\jmath)$ and $t \in[0,1]$ then

$$
\left|a(\jmath, t)_{1}-b(J, t)_{1}\right|>2^{2-k} \Rightarrow\left|a(J, t)_{t+n(t)}-a(\jmath, t)_{1}\right| \leq 2^{2-t}
$$

for all $J-1 \geq l \geq k$

Thus conditions $(1)_{j+1},(111)_{j}$ and (iv), come over from Lemma 31 unchanged, conditions (11), and (v) ${ }_{j+1}$ are strengthened whilst conditions (1v), (vil), and (vi11), are new A proof of Lemma 41 will thus give a proof of Lemma 31 The first step in the inductive proof of Lemma 41 is simple

LEMMA 42 Let $n(1)=m(1)=5, N(1)=3$ and

$$
\begin{array}{lc}
a(1, t)=(0,0, t, 0,0) & (0 \leq t \leq 1) \\
b(1, t)=(0,0,-t, 0,0) & (0 \leq t \leq 1) \\
c(1, t)=(0,0,0,0,0) & (0 \leq t \leq 1)
\end{array}
$$

Then conditions $(\mathrm{n})_{1},(111)_{1},\left(\mathrm{v}_{1}\right)_{1},(\mathrm{v} 11)_{1}$ and $(\mathrm{ix})_{1}$ of Lemma 41 are sattsfied

Proof Direct inspection (Condition $(1 \mathrm{x})_{1}$ is vacuously satisfied)

To complete the induction we use the following lemma (in which an attempt has been made to simplify the notation)

Lemma 43 Suppose $n(J) \geq 5 n(l)$ for all $J-1 \geq l \geq 1$ and suppose that the three functions from $[0,1]$ to $[-1,1]^{n(J)}$ whose values at $0 \leq t \leq 1$, given by $a(t)=a(j, t)$, $b(t)=b(J, t), c(t)=c(J, t)$, satisfy conditions $(11)_{J},(111)_{J},(\mathrm{v})_{j},(\mathrm{v1})_{J},(\mathrm{v} 11)_{j}$, and $(\mathrm{ix})_{J}$ of Lemma 41 Then we can find $m(j+1) \geq 5$ and three functions from $[0,1]$ to $[-1,1]^{n(J+1)}$ (where $n(J+1)=n(J) m(j+1)$ ) whose values at $0 \leq t \leq 1$ are given $b_{1}$ $A(t)=a(\jmath+1, t), B(t)=b(J+1, t), C(t)=c(\jmath+1, t)$ and satisfy all the conditions

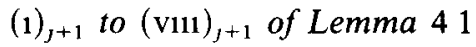

Proof Since $a, b, c$ are continuous on $[0,1]$ they are uniformly contınuous We can therefore find an $\eta>0$ such that $\eta^{-1}$ is an integer and $\left|a(t)_{r}-a(\tau)_{r}\right|,\left|b(t)_{r}-b(\tau)_{r}\right|$, $\left|c(t)_{r}-c(\tau)_{r}\right|<2^{-\jmath-1}$ whenever $|t-\tau| \leq \eta$ and $1 \leq r \leq n(\jmath)$ We set $M=\eta^{-1}+1$, $m(J+1)=18 M+n(J)$ and define $A, B$ and $C$ as follows

If $0 \leq l \leq 15 M, 1 \leq r \leq n(j)$ we set

$$
A(t)_{\ln (\jmath)+r}=B(t)_{\ln (\jmath)+r}=C(t)_{\ln (\jmath)+r}=E_{\ln (\jmath)+r}
$$

where

$$
\begin{aligned}
E_{l n(j)+r} & =a(l \eta)_{r} & & (0 \leq l \leq M) \\
& =a(1)_{r} & & (M+1 \leq l \leq 4 M) \\
& =a((5 M-l) \eta)_{r} & & (4 M+1 \leq l \leq 5 M) \\
& =b((l-5 M-1) \eta)_{r} & & (5 M+1 \leq l \leq 6 M) \\
& =b(1)_{r} & & (6 M+1 \leq l \leq 9 M) \\
& =b((10 M-l) \eta)_{r} & & (9 M+1 \leq l \leq 10 M) \\
& =c\left((l-10 M-1)_{\eta}\right)_{r} & & (10 M+1 \leq l \leq 11 M) \\
& =c(1) & & (11 M+1 \leq l \leq 14 M) \\
& =c((15 M-l) \eta)_{r} & & (14 M+1 \leq l \leq 15 M)
\end{aligned}
$$


If $15 M+1 \leq l \leq 16 M, 1 \leq r \leq n(\jmath)$ we set

$$
\begin{aligned}
& A(t)_{\ln (\jmath)+r}=C(t)_{\ln (\jmath)+r}=a((l-15 M-1) \eta t)_{r} \\
& B(t)_{\ln (\jmath++r}=b((l-15 M-1) \eta t)_{r},
\end{aligned}
$$

whilst if $16 M+1 \leq l \leq 17 M, 1 \leq r \leq n(j)$ we set

$$
\begin{aligned}
& A(t)_{\ln (\jmath)+r}=a((17 M-l) \eta t)_{r} \\
& B(t)_{\ln (\jmath)+r}=C(t)_{l n(\jmath)+r}=b((17 M-l) \eta t)_{r}
\end{aligned}
$$

It is easy to check that conditions (1v) $)_{j+1},(\mathrm{v})_{j+1}$ and $(\mathrm{v} 1)_{j+1}$ are satisfied and to see that condition $\left(\mathrm{v}_{11}\right)_{\jmath+1}$ is satisfied it suffices to take $N(J+1)=16 M n(J)+N(J)$ We check the remainıng conditions $(1)_{j+1},(11)_{j+1},(111)_{j+1}$ and $(v 111)_{j+1}$ one by one

Condition $(1)_{j+1}$ Suppose $u \in U(J)$ and $v \in U(j+1)$ Then $u \in\{a(t), b(t), c(t)\}$ for some fixed $0 \leq t \leq 1$ Choose $1 \leq k \leq M$ so that $|(k-1) \eta-t|<\eta$ By the choice of $\eta$

$$
\begin{aligned}
\left|E_{k n(\jmath)+r}-a(t)_{r}\right| & =\left|a((k-1) \eta)_{r}-a(t)_{r}\right|<2^{-\jmath-1}, \\
\left|E_{(5 M+k) n(\jmath)+r}-b(t)_{r}\right| & =\left|b((k-1) \eta)_{r}-b(t)_{r}\right|<2^{-\jmath-1}, \\
\left|E_{(10 M+k) n(\jmath)+r}-c(t)_{r}\right| & =\left|c((k-1) \eta)_{r}-c(t)_{r}\right|<2^{-\jmath-1},
\end{aligned}
$$

for all $1 \leq r \leq n(J)$ It follows that

$$
\min _{0 \leq p \leq 2} \max _{1 \leq r \leq n(\jmath)}\left|v_{(5 P M+k) n(J)+r}-u_{r}\right|<2^{-\jmath-1}
$$

and condition $(1)_{j+1}$ follows

Condition $(11)_{1+1}$ We begin with two simplifying remarks Firstly since $u_{r+n(j+1)}=u_{r}$ the condition $1 \leq r, s \leq 2 n(J)$ may be ignored Secondly if $r-s=m$ then $n(J+1)+s-r=n(J+1)-m$ and so we need only consider $1 \leq m \leq n(j+1) / 2+1$ Even so, we shall distınguish 3 cases according as $1 \leq m \leq M n(J)$ and $m \neq 0 \bmod n(J), 1 \leq m \leq M n(J)$ and $m \equiv 0 \bmod n(J)$, or $M n(J)+1 \leq m$

If $1 \leq m \leq M n(J)$ and $m \neq 0 \bmod n(J)$ then $m=k n(J)+\mu$ for some integers $1 \leq k \leq$ $M$ and $1 \leq \mu \leq n(J)$ By condition (11), we know that there exist integers $1 \leq \rho$, $\sigma \leq 2 n(J)$ such that

It follows that

$$
\max \left(\left|a(1)_{\rho}-a(1)_{\sigma}\right|,\left|b(1)_{\rho}-b(1)_{\sigma}\right|,\left|c(1)_{\rho}-c(1)_{\sigma}\right|\right) \geq 1
$$

$$
\max _{0 \leq p \leq 2}\left(\left|C(1)_{((5 p+1) M+k) n(\jmath)+\mu}-C(1)_{(5 p+1) M n(\jmath)+\sigma}\right|\right) \geq 1
$$

We note that

$$
(((5 P+1) M+k) n(\jmath)+\mu)-((5 P+1) M n(J)+\sigma)=m,
$$

so our discussion of this case is complete

If $1 \leq m \leq M n(J)$ and $m \equiv 0 \bmod n(J)$ then $m=k n(J)$ for some $1 \leq k \leq M$ Thus settıng $r=(16 M+k) n(J)+N(J)$ and $s=16 M n(J)+N(J)$ we have $r-s=m$ and, using condition (vi1),

$$
\left|C(1)_{r}-C(1)_{\triangleleft}\right|=16((M-k) \eta)_{N(j)}-a(1)_{N(j)} \mid
$$

Finally, if $M n(J)+1 \leq m \leq n(J+1) / 2+1$ then takıng $r=16 M n(J)+N(J)$ and $s=$ $r-m$ we have $1 \leq s \leq(15 M+1) n(\jmath)$ and so $A(1)_{s}=C(1)_{s}$ whilst $A(1)_{r}=1, C(1)_{r}=$ -1 Thus

$$
\left|A(1)_{r}-A(1)_{s}\right|+\left|C(1)_{r}-C(1)_{s}\right|=\left|1-A(1)_{s}\right|+\left|A\left(1_{s}\right)+1\right| \geq 2
$$


and so $\max \left(\left|A(1)_{r}-A(1)_{s}\right|,\left|C(1)_{r}-C(1)_{s}\right|\right) \geq 1$ Combining the 3 cases discussed we obtain condition (11) $)_{j+1}$

Condition (111) $)_{j+1}$ Observe first that this condition is vacuously satisfied if $J=1$ We may therefore suppose $\jmath \geq 2$ We distinguish 2 cases according as $p \leq \jmath-1$ or $p=\jmath$ If $p \leq J-1$ the argument is exactly the same as that used to establish condition (111) in the proof of Lemma 32 from Lemma 31 If $p=J$ we argue as follows

By the choice of $\eta$ and the definitions of $A, B$ and $C$

$$
\left|A(t)_{1}-A(t)_{\imath+n(\jmath)}\right|,\left|B(t)_{\imath}-B(t)_{\imath+n(\jmath)}\right|<2^{-\jmath-1}
$$

for all $1 \leq \imath \leq n(\jmath+1)$ whilst

$$
\left|C(t)_{\imath}-C(t)_{\imath+n(\jmath)}\right|<2^{-\jmath-1}
$$

for all $1 \leq \imath \leq(16 M-1) n(\jmath)$ and all $16 M n(\jmath)+1 \leq \imath \leq n(\jmath+1)(0 \leq t \leq 1)$ Further, by $(\mathrm{v})_{j}$,

$$
C(t)_{16 M n(J)-n(J-1)+r}=c(J-1,0)_{r}=C(t)_{16 M n(J)+n(J)-n(J-1)+r}
$$

for all $1 \leq r \leq n(j-1)$ and so

$$
\left|C(t)_{t}-C(t)_{t+n(j)}\right|=0<2^{-\jmath-1}
$$

for all $16 M n(J)-n(J-1)+1 \leq \imath \leq 16 M n(\jmath)$ Thus if $1 \leq \imath \leq n(\jmath+1)$ and $\left|u_{t}-u_{t+n(j)}\right|>2^{2-k}$ we can conclude that $u=C(\tau)$ for some $0 \leq \tau \leq 1$ and $\imath=(16 M-1) n(\jmath)+q$ for some $1 \leq q \leq n(\jmath)-n(J-1)$

It follows that $u_{i}=C(\tau)_{l}=a(\tau)_{q}$ and $u_{1+n(J)}=C(\tau)_{\imath+n(\jmath)}=b(\tau)_{q}$ Since $\left|a(\tau)_{q}-b(\tau)_{q}\right|>2^{2-k}$ we see from (v111), that $\left|a(\tau)_{q+n(l)}\right| \leq 2^{2-1}$ and so, since $a(\tau)_{q+n(l)}=C(\tau)_{l+n(l)}=u_{i+n(l)}$, that $\left|u_{\imath}-u_{i+n(l)}\right| \leq 2^{2-l}$ for all $J-1 \geq l \geq k$ as required

Condition (v111) ${ }_{\jmath+1}$ As we observed above, $\left|A(t)_{t}-A(t)_{\imath+n(\jmath)}\right|<2^{-\jmath-1}$ for all $1 \leq t \leq$ $n(j+1)$ and all $0 \leq t \leq 1$ Thus we need only check condition (vi11) $)_{j+1}$ for $j-1 \geq l \geq k$ since the case $l=J$ is settled automatically Since when $J=1$ there are no further values of $l$ to consider we may suppose $j \geq 2$

By construction $A(t)_{1}=B(t)_{\imath}$ for all $1 \leq t \leq 15 M n(J)$ and, by condition (vi), $A(t)_{1}=B(t)_{1}$ for all $P n(J)-n(j-1)+1 \leq l \leq P n(J)$ Thus if $\left|A(t)_{l}-B(t)_{1}\right|>2^{2-k}$ it follows that $\imath=k n(\jmath)+r$ where $15 M \leq k \leq 17 M-1$ and $1 \leq r \leq n(\jmath)-n(J-1)$, and so $A(t)_{t}=a(\tau)_{r}, B(t)_{l}=b(\tau)_{r}$ for some $1 \geq \tau \geq 0$ We now have $\left|a(\tau)_{r}-b(\tau)_{r}\right|>2^{2-k}$ whence, by condition (vili),,$\left|a(\tau)_{r+n(l)}-a(\tau)_{r}\right| \leq 2^{2-l}$ for all $j-1 \geq l \geq k$ Since $a(\tau)_{r+n(l)}=A(t)_{i+n(l)}$ this yields $\left|A(t)_{i+n(l)}-A(t)_{l}\right| \leq 2^{2-l}$ for all $J-1 \geq l \geq k$ as required

Lemmas 42 and 43 together give Lemma 41 and so the proof is complete

\section{REFERENCE}

[1] H Furstenberg Recurrence in ergodic theory and combinatorial number theory Princeton 1981 